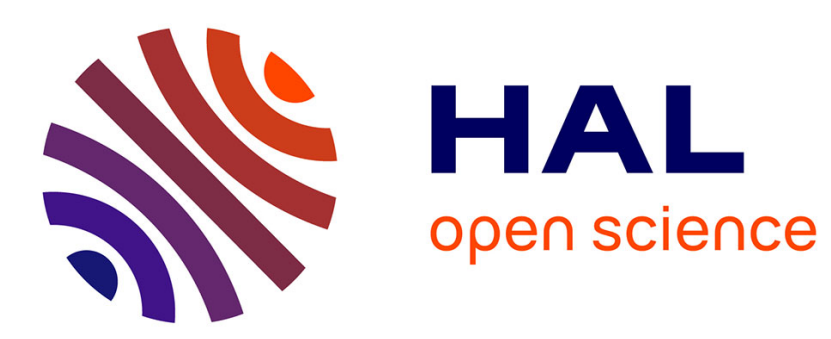

\title{
Approche exploratoire du discours autour de la suspension dans le cadre du body art
}

D. Rochaix, A. Bonnet, Jean-Louis Pedinielli

\section{To cite this version:}

D. Rochaix, A. Bonnet, Jean-Louis Pedinielli. Approche exploratoire du discours autour de la suspension dans le cadre du body art. Annales Médico-Psychologiques, Revue Psychiatrique, 2011, 169 (5), pp.292. 10.1016/j.amp.2010.11.014 . hal-00765609

\section{HAL Id: hal-00765609 \\ https://hal.science/hal-00765609}

Submitted on 15 Dec 2012

HAL is a multi-disciplinary open access archive for the deposit and dissemination of scientific research documents, whether they are published or not. The documents may come from teaching and research institutions in France or abroad, or from public or private research centers.
L'archive ouverte pluridisciplinaire HAL, est destinée au dépôt et à la diffusion de documents scientifiques de niveau recherche, publiés ou non, émanant des établissements d'enseignement et de recherche français ou étrangers, des laboratoires publics ou privés. 


\section{Accepted Manuscript}

Title: Approche exploratoire du discours autour de la suspension dans le cadre du body art

Authors: D. Rochaix, A. Bonnet, J.-L. Pedinielli

PII: $\quad$ S0003-4487(11)00060-6

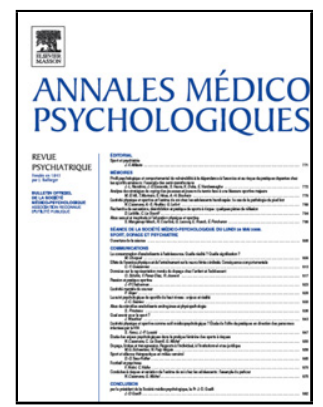

DOI: $\quad$ doi:10.1016/j.amp.2010.11.014

Reference: $\quad$ AMEPSY 1304

To appear in: $\quad$ Annales Médico-Psychologiques

Received date: $\quad 2-8-2010$

Accepted date: $\quad 12-11-2010$

Please cite this article as: Rochaix D, Bonnet A, Pedinielli J-L, Approche exploratoire du discours autour de la suspension dans le cadre du body art, Annales mediopsychologiques (2010), doi:10.1016/j.amp.2010.11.014

This is a PDF file of an unedited manuscript that has been accepted for publication. As a service to our customers we are providing this early version of the manuscript. The manuscript will undergo copyediting, typesetting, and review of the resulting proof before it is published in its final form. Please note that during the production process errors may be discovered which could affect the content, and all legal disclaimers that apply to the journal pertain. 


\title{
Mémoire
}

Approche exploratoire du discours autour de la suspension dans le cadre du body art Exploratory approach of speech about suspension in the context of body art D. Rochaix ${ }^{\text {a }}$, A. Bonnet ${ }^{\text {b }}$, J.-L. Pedinielli ${ }^{\text {b }}$

${ }^{a}$ Centre PsyCLE EA3273, UFR psychologie, UFR de psychologie, Université Aix-Marseille, 29, avenue Schuman, 13621 Aix-en-Provence cedex 1, France

${ }^{b}$ Laboratoire de Psychopathologie Clinique et de Psychanalyse EA 3278, UFR de psychologie, Université Aix-Marseille, 29, avenue Schuman, 13621 Aix-en-Provence cedex 1, France

Auteur correspondant: D. Rochaix, Centre PsyCLE EA3273, UFR psychologie, UFR de psychologie, Université Aix-Marseille, 29, avenue Schuman, 13621 Aix-en-Provence cedex 1, France

Adresse email : delphine.rochaix@ hotmail.fr

Texte reçu le 5 août 2010 ; accepté le 12 novembre 2010

\section{Résumé}

Cette analyse sémantique menée à l'aide d'un logiciel d'analyse de discours (Tropes) propose une réflexion sur le discours d'un sujet portant sur une pratique du Body Art : la suspension. Il s'agit d'une recherche exploratoire présentant une analyse d'un texte écrit par un jeune homme pratiquant la suspension. Le but étant de se centrer sur le discours porté sur l'acte de suspension.

Mots clés : Analyse sémantique ; Body Art ; Hypothèses psychopathologiques ; Suspension

\begin{abstract}
This semantic analysis led by a discourse analysis software (Tropes) proposes a reflection concerning a speech about Body Art's practice: suspension. It is an exploratory research presenting an analysis of a text written by a young man practicing the suspension. The purpose being to focus on the speech concerned the act of suspension.
\end{abstract}

Keywords: Body Art; Psychopathological hypothesis; Semantic analysis; Suspension 


\section{Introduction}

Le Body Art, ou art corporel, constitue un large domaine faisant appel à diverses dimensions : artistique, sociétale, culturelle et psychologique. Il englobe les performances artistiques (play piercing, suspension) et les actes de modifications corporelles (piercing, implant, tatouage). Par-delà l'approche esthétique, on peut d'un point de vue psychopathologique rapprocher les pratiques du Body Art des conduites à risque, des tendances perverses, et envisager ces comportements comme sous-tendus par des types de personnalité, de fonctionnement psychique, ainsi que par des mécanismes particuliers.

Les pratiques issues du Body Art évoquent les conduites à risque dont Bonnet et al. [1] rappellent les caractéristiques, positives d'expérimentation, et négatives renvoyant aux aspects destructeurs. Ici, l'intégrité physique est notamment mise en jeu par la recherche de dépassement de soi et des limites physiques. L'investissement massif du corps par l'effort rappelle de plus les pratiques sportives dans lesquelles la répétition est présente. Selon Pedinielli et Bonnet [2,13], la notion de répétition est à questionner sous l'angle de la recherche d'un état antérieur, ou comme étant le résultat du comportement inhérent à la dépendance physique et/ou psychologique. L'analogie entre l'utilisation du corps dans l'Art et l'apparition de nouveaux symptômes relatifs aux atteintes corporelles est appréhendée par certains auteurs [6,12]. Cela nous amène à nous questionner sur les caractéristiques communes aux dimensions artistiques et pathologiques, dont les limites apparaissent floues.

\section{Caractéristiques du body art}

Évoquer le Body Art nécessite de faire un détour par le courant turbulent des années 1960 qui fut son précurseur. C'est de ce passé culturel et historique que ce mouvement tire le caractère engagé de certaines de ses œuvres. L'Actionnisme viennois a en effet établi les bases de ce courant en Autriche par les actions/happenings (ou «art en train de se faire ») de Nitsch, Brus, Muehl, ou encore Schwartzkogler. Le contexte social d'après-guerre de l'époque fait que la visée de cet art est davantage sociale qu'esthétique [10]. Cette dimension se retrouve de nos jours : certains artistes ont défendu ou défendent ainsi par leurs pratiques la condition féminine (Gina Pane), le droit d'utiliser son corps librement (Orlan, Stelarc), d'autres prônent l'expérimentation (Fakir Musafar), ou encore mettent en scène des éléments intimes et porteurs de sens plus ou moins cachés. Selon Schäfer [14], « Le tableau devient un 
psychogramme, un document de l'affect » qu'il paraît intéressant d'analyser et de lire, sans le stigmatiser toutefois, sur un versant psychopathologique.

En outre, le courant du Body Art présente la caractéristique de fondre l'œuvre/la création avec son auteur. Le corps est le matériau. «La peau à la place de la toile, mais aussi les liquides du corps à la place de la peinture, le bistouri du chirurgien à la place du pinceau ou du burin du sculpteur »[7]. Le spectateur se trouve alors dans une autre dynamique : il n'observe plus une œuvre / une performance, mais l'artiste lui-même.

Le Body Art renvoie de plus à la modification corporelle (ou bodmod, contraction pour body modification), désignant alors des individus sous le terme de bodmoders, terme faisant à la fois référence à la mode, à l'art et à la performance. Cette dernière définit les actions réalisées dans lesquelles le corps est mis à l'épreuve sous forme de défi et d'expérimentation. Il s'agit pour certains individus d'endurer une épreuve, de modifier leur corps, d'y inscrire quelque chose en lien avec un événement clé de leur vie [3], inscription destinée à eux-mêmes ou à un autre. Les pratiques sont nombreuses, mais nous traiterons ici de la suspension. La suspension consiste à insérer des crochets sous la peau, à différents emplacements sur le corps (dos, genou, bras) en les répartissant de telle sorte que les zones corporelles transpercées puissent supporter tout le poids de l'individu. L'étape suivante consiste à élever le corps à l'aide de câbles passant dans les crochets. Ce type de performance est réalisé devant un public (au sein d'un cadre relativement privé possédant une dimension initiatique, ou plus ouvert lors de manifestation culturelle, concert), cet acte étant la plupart du temps médiatisé. Photographies, vidéos sont en effet diffusées sur Internet, seul vestige ou presque de ces œuvres éphémères (en faisant exception des cicatrices). La suspension se situe en effet audelà de l'ornementation corporelle dont relèvent entre autres le piercing ou le tatouage, puisque ici, il ne reste rien à montrer en apparence, rien à porter sur sa peau une fois les crochets retirés.

Le choix d'étudier la suspension découle en premier lieu du caractère relativement nouveau de cette pratique et de son approche, récente et encore rare, en psychopathologie. En second lieu, nous avons pu constater, au cours d'une recherche exploratoire, que la douleur n'était pas, selon les dires des sujets, ressentie lors de la suspension. Il est alors intéressant de questionner ce type de pratique d'allure violente et, paradoxalement, semblant indolore ; cela peut venir pointer par exemple un décalage entre ce que dit le sujet et ce qu'il ressent effectivement, ou encore laisser entendre à une minimisation de la douleur provoquée par la présence de bénéfices (la jouissance masochiste inconsciente). Selon Lauru [8], « la peau parle l'état du corps [...]», aussi la tentative de comprendre ce qui sous-tend ces actes qui 
abîment/transpercent/étirent la peau paraît-elle importante. Cela d'autant plus, peut-être, qu'ils prennent, à l'heure actuelle, une ampleur significative dans notre société.

\section{Objectifs}

Le but de la recherche est d'étudier, à partir d'une analyse de discours, le rapport du sujet à l'acte de suspension et de repérer les éléments saillants du texte d'un point de vue sémantique.

\section{Méthodologie}

\subsection{Présentation du matériel à analyser}

Ce fragment de texte est celui d'un sujet inscrit dans le Body Art ayant participé à la recherche exploratoire sur ce thème. Les sujets de l'échantillon de recherche ont répondu à un questionnaire visant à décrire leurs pratiques et à développer en quelques lignes une pratique privilégiée ayant trait au Body Art. La majorité des participants se sont prêtés à cet exercice en évoquant rapidement leurs pratiques. Le sujet dont nous avons analysé le discours a, en revanche, produit un véritable texte pour décrire une séance de suspension. La description qu'il a faite de cette pratique s'est distinguée de celles que les autres participants ont pu réaliser, notamment par la richesse des détails évoqués ainsi que par la longueur du paragraphe élaboré.

\subsection{Traitement des données}

Nous avons procédé à une analyse sémantique à l'aide du logiciel Tropes (Zoom VF7). L'utilisation de ce type de logiciel permet d'avoir une analyse qualitative objective d'un texte en le découpant en différentes catégories selon le lexique employé par l'auteur (verbes, connecteurs, modalisations). Les mots du texte sont répartis dans ces différentes catégories, permettant alors d'étudier la manière dont ils se rapprochent, se succèdent, ainsi que leur emplacement dans le corps du texte. Cela nous renseigne sur le style dans lequel le texte est écrit, par extension sur l'implication du sujet ; sur la fréquence d'apparition des mots dans le texte et les univers de références auxquels ils renvoient. 


\section{Analyse du texte}

\subsection{Présentation du sujet}

L'auteur du texte, nous l'appellerons Sébastien, est un jeune homme de 20 ans, ayant accepté de participer à une recherche sur le thème du Body Art. À la question portant sur la description brève de leur première pratique en lien avec ce courant, Sébastien choisit de décrire la suspension, qu'il présente de la manière suivante : «La suspension consiste à se pendre par des crochets situés à un endroit choisi de son corps. Le but en est un dépassement de soi-même ainsi que la sensation des endorphines s'affolant dans son corps. » Nous présentons ainsi certains passages du texte tels qu'il les a rédigés.

\subsection{Présentation des résultats : analyse sémantique}

Le texte renvoie à un découpage que l'on peut opérer concernant l'acte en lui-même, mais aussi les fonctions des étapes qui le constituent. La temporalité de l'acte semble s'étendre à la construction du texte que Sébastien présente de façon chronologique. Nous percevons donc plusieurs épisodes qui, bien que non équivalents quantitativement, serviront à découper le texte pour mener l'analyse :

- la mise en place (arrivée sur les lieux de Sébastien, prise de contact avec les individus présents, insertion des crochets). Ce fragment de texte comprend approximativement 469 caractères. La mise en place englobe la préparation psychique et la préparation matérielle. Il s'agit alors pour le sujet de prévoir l'emplacement de son corps qui sera transpercé, le lieu et le moment de la suspension, ainsi que les personnes qui vont procéder à l'intervention. De plus, Sébastien présente l'insertion des crochets comme un préliminaire à la suspension, renvoyant à une minimisation de cette étape, ou au contraire à un investissement particulier de la suspension en tant que telle ;

- l'action (la suspension). Ce fragment de texte comprend approximativement 798 caractères. Sébastien décrit les différentes phases d'habituation par lesquelles il passe pour se livrer à la suspension. Le corps est en effet élevé graduellement, à son signal et après différents «breaks»;

- l'après coup (contact avec les individus). Ce fragment de texte comprend approximativement 416 caractères. Le sujet décrit le moment où il regagne le sol : «au 
moment ou j'atteins le sol c'est comme si je pesais $300 \mathrm{~kg}$, je m'écrase par terre », puis il revient sur le caractère positif de cette expérience.

Concernant la trame du récit, Sébastien produit son texte par paliers. Il décrit les préparatifs de la suspension, l'acte en lui-même, et l'instant qui suit.

\subsubsection{Présentation des caractéristiques du texte : tableau 1}

Le texte est écrit dans un style argumentatif, avec une prise en charge par le « je », signant une implication du sujet dans son récit. Sébastien parle de son expérience propre, il ne généralise pas la suspension et ne semble pas se servir du recours à la norme dans son langage. La mise en scène est dynamique et se situe dans l'action. Globalement, nous constatons que le sujet s'implique dans son discours par l'utilisation majoritaire de pronoms personnels «je» $(74,5 \%)$. Toutefois, nous constatons l'utilisation du pronom « on» $(13,7 \%)$, relativement bien ciblée dans le texte puisqu'elle renvoie aux deux premières phases (mise en place et action : « on accroche », « on met un peu de tension », « on commence à lever »). Sur l'ensemble du texte, les verbes employés sont principalement des verbes factifs $(62,4 \%)$, qui renvoient à des actions, puis des verbes statifs $(30,1 \%)$ qui expriment ici des états. Le sujet utilise des modalisations d'intensité (40,5\%) qui dramatisent le discours, tout comme les modalisations de négation, présentes ici en proportion moindre (13,5\%). Des modalisations de temps $(21,6 \%)$ et de manière $(16,2 \%)$ sont également présentes, permettant de situer son récit.

\subsubsection{Présentation des épisodes : tableau 2}

Les épisodes se révèlent homogènes et présentent une mise en scène dynamique menée par l'action. Nous notons que le style de l'épisode 1 (mise en place) est plutôt énonciatif, contrairement aux deux autres épisodes où il ressort un style plutôt argumentatif. Dans les deux derniers épisodes, le sujet emploie des termes destinés à articuler son discours et présenter des liens logiques entre ses idées (mais, car, donc), de sorte à argumenter, voire à convaincre, montrant par son écriture qu'il est acteur de sa suspension.

\subsubsection{Caractéristiques des épisodes : tableau 3}


Les verbes factifs et statifs se retrouvent majoritairement dans l'épisode 2 (action). Nous nous interrogeons sur le statut de l'acte et nous pouvons observer un double mouvement de banalisation et d'exagération dans la façon de présenter la suspension, plus précisément l'insertion des crochets. Sébastien évoque cette phase : «niveau sensation, franchement le passage des crochets, ça casse pas des briques, c'est même plutôt sympa ", l'expression émotionnelle étant relativement impersonnelle. Les modalisations d'intensité employées dans l'ensemble du texte et en majorité dans les phases un et deux (mise en place, action) vont également dans le sens d'une banalisation («ça brûle un peu »). Pourtant, l'analyse du fragment de texte relatif à l'insertion des crochets révèle une importante utilisation de modalisations d'intensité. En parallèle, il accentue la performance, « après m'être fait éponger le sang qui coule à flot». Par ailleurs, il emploie le terme «papoter», terme léger qui introduit l'acte (il papote avant sa suspension) et le clôture (il papote après), donnant un effet de légèreté à l'acte de suspension.

\subsubsection{Présentation des thèmes abordés : tableau 4}

Les univers de références permettent de présenter le contexte en indiquant les termes les plus fréquents dans le texte (noms communs et noms propres). Cela nous renseigne globalement sur le contenu du texte et permet d'avoir accès aux représentations de l'auteur quant à la suspension. L'univers de référence 1 présente les différentes thématiques du discours, dans le contexte général de leur apparition; l'univers 2 propose un contexte plus précis qui offre un découpage plus fin du lexique employé. Ces univers de références s'apparentent à des résumés, des fiches de lecture qui renseignent rapidement sur la teneur du texte et amorcent d'ores et déjà l'analyse sémantique. Ainsi, nous pouvons constater que le récit suit une trame qui renvoie à différentes thématiques.

Ainsi, l'épisode 1 (mise en place) pose le cadre dans lequel va se jouer la suspension. La dimension temporelle est présente, laissant penser que ces actes se situent bien dans un cadre de temps et d'espace particulier. On perçoit la référence aux «gens » et à la «photographie » comme des éléments importants, quasiment incontournables. La notion de sensation émerge lors de l'insertion des crochets, ou plutôt l'absence de sensation. La notion de plaisir est également évoquée lorsque Sébastien présente la suspension et la libération d'endorphine dans son organisme. Cette stimulation entraîne une réaction chimique par libération d'hormones dont les propriétés présentent la particularité d'être à la fois antalgiques 
et précurseurs de plaisir. On peut d'ailleurs se questionner sur l'efficacité analgésique de l'endorphine, ainsi que le lien pouvant exister entre douleur et plaisir.

L'épisode 2 (la suspension) évoque un temps qui paraît en suspens et des éléments clivés. Il semble y avoir une opposition tête/corps (peau, pied), terre/pied, une distinction entre le corps suspendu (hors limite) et le corps ancré au sol. Le corps se positionne par rapport à l'espace, renvoyant à un clivage : un corps dans l'espace et un corps hors de l'espace. On observe le même mouvement au niveau du cadre temporel. Le corps est inscrit dans une certaine temporalité et demeure lié à la réalité externe lorsque les pieds du sujet touchent le sol. Puis, il semble être en dehors du temps lorsqu'il est suspendu, hors limite de par la dimension d'irréalité de la position de suspension. Nous pouvons de plus établir un parallèle avec la description d'une première expérience sexuelle. Cette dimension sexuelle se retrouve en effet à la fois dans les termes utilisés par Sébastien et dans la dimension symbolique que le texte peut évoquer. On relève également dans une de ses dernières tournures «plutôt satisfaite d'avoir tenu 25 minutes sans problème pour une première » qu'en plus de la faute de frappe, la performance sexuelle est une fois de plus évoquée. L'expression émotionnelle est, en outre, imagée, peut-être impersonnelle : «J'ai pris mon pied, ça casse pas des briques, comme si je pesais $300 \mathrm{~kg}$. » Le style du texte tient lieu, selon Zapata-Reinert [15], « de symptôme dans le registre du discours ». Nous pouvons nous questionner sur l'utilisation de figures de styles ou de formules imagées pour décrire des émotions, soustendues peut-être par des difficultés pour Sébastien à exprimer ce qu'il ressent.

L'épisode 3 (l'après-coup), où le corps rejoint son élément lourdement, comme si l'expérience d'intemporalité prenait fin. La référence aux individus/spectateurs est visible, leur présence garantissant peut-être au sujet le maintien d'un lien avec la norme rassurante.

\section{Discussion}

Il paraît intéressant de pallier les limites de cette approche en envisageant une prochaine étude plus conséquente en termes d'échantillon et de production des participants. Ce texte, bien qu'il soit assez court, propose quelques grilles de lecture de cette performance. La suspension vient s'inscrire dans ces pratiques actuelles qui se situent à la limite de l'exhibition, du voyeurisme, du sadisme, du masochisme, et dans lesquelles les places apparaissent comme interchangeables. Le corps demeure l'invariant, nous nous interrogeons sur la place qu'il occupe. Nous questionnons également les intentions de l'auteur du texte sur ce qu'il souhaite déclencher chez son lecteur. La lecture semble s'inscrire, peut-être, comme 
la photographie ou la vidéo, dans la continuité de l'acte de suspension. Tout se passe comme si l'acte était optimisé pour fournir le plus de plaisir possible. Ainsi, il se découpe en plusieurs épisodes (mise en place, acte, après-coup), renvoyant chacun à des bénéfices particuliers (fantasme/scénario, décharge, fantasme/voyeurisme-exhibitionnisme). Nous interrogeons la question du bénéfice sur le versant du détournement d'affects pénibles par l'investissement et la maîtrise du corps, ou du plaisir (là encore multiple : plaisir masochiste, exhibitionniste, toute-puissance). Il semble vouloir bousculer le spectateur. L'autre, le public, fait partie intégrante des performances qui n'existeraient pas sans lui. De plus, ces pratiques demeurent relativement bien intégrées socialement (elles sont menées sans retenue ou presque devant un public averti, ou sont « déguisées », dissimulées derrière la dimension de spectacle face à un public plus large). Lebrun [9] évoque une «économie collective perverse », correspondant à un déni massif de la castration. Elle ne signe pas un fonctionnement individuel pervers mais bien une «nouvelle économie psychique » qui accompagne l'évolution des mœurs et qui prône la jouissance plutôt que le désir [11], l'expérimentation et la découverte de bénéfices nouveaux étant poussées à l'extrême. Reste à savoir si c'est le scénario de l'individu qui suspend qui est appliqué ou celui de l'individu suspendu ? L'analyse d'un écrit ne peut être aussi complète qu'une analyse de discours. Cette démarche aurait été pertinente par l'appréhension de ce qui accompagne le discours (signes verbaux qui traduisent par exemple une exagération, ou signes non verbaux orientant vers une altération de la réalité) et l'étude de la congruence entre le discours et l'attitude [4]. Kaplan et Grabe [5] rappellent, par ailleurs, la nécessité de considérer les intentions et la démarche de l'auteur qui rédige un texte vis-à-vis du lecteur. Nous avons enfin conscience, ici, de la difficulté que cette tâche a pu présenter pour Sébastien qui a dû produire un texte sans pouvoir bénéficier d'un étayage de l'interlocuteur, comme c'est le cas dans un entretien.

\section{Conclusion}

Il est intéressant de questionner les pratiques de cette population qui a priori ne consulte pas de psychologue mais accepte de se livrer dans le cadre d'une recherche. Les actes de suspension prennent de l'ampleur dans notre société actuellement, et semblent relativement acceptés. Tantôt présentés comme œuvre artistique ou rituel initiatique, tantôt comme actes déviants, il s'avère en tout cas qu'il est possible de les pratiquer, qui plus est en public. Il y a donc là un double mouvement autour de ce courant qui est selon le point de vue 
adopté, valorisé, ou du moins toléré, dans un autre cas de figure proscrit ou synonyme de trouble psychologique.

\section{Conflit d'intérêt : aucun}

\section{Références}

[1] Bonnet A, Pedinielli JL, Rouan G. Bien-être subjectif et régulation émotionnelle dans les conduites de risques. Le cas de la plongée sous-marine. L'Encéphale 2003;29:488-95.

[2] Bonnet A, Bréjard V. Addictions à l'activité physique. In: Varescon, I. Les addictions comportementales. Paris: Mardaga; 2009. p.237-266.

[3] Grognard C. Tatouage, piercing : décoration ? Décorporation ? Dénaturation du corps ou retour au primitif ? Gynécologie Obstétrique \& Fertilité 2006;34:41-3.

[4] Jonas C. Signes physiques et verbaux du mensonge : résultats des études américaines. Ann Méd Psychol 2007;165:369-71.

[5] Kaplan RB, Grabe W. A modern hystory of written discourse analysis. Journal of second language writing 2002;11:191-223.

[6] Korff-Sausse S. Les corps extrêmes dans l'art contemporain. Entre perversion et créativité. Champ Psychosomatique 2004;35:61-74.

[7] Korff-Sausse S. Quelques réflexions psychanalytiques sur le Body Art. Champ Psychosomatique 2004;36:171-83.

[8] Lauru D. Perçons corps. Champ Psychosomatique 2004;36:119-29.

[9] Lebrun JP. La perversion ordinaire, vivre ensemble sans autrui. Paris: Denoël; 2007.

[10] Lussac O. Fluxus et propagande politique : des buts sociaux, non esthétiques. Actuel Marx 2002;32:169-83.

[11] Melman C. L'homme sans gravité. Paris: Denoël; 2002.

[12] Merot P. Art corporel : le corps entre pensée sublimatoire et pensée opératoire. Revue française de psychanalyse 2005;695:1583-96.

[13] Pedinielli JL, Bonnet A. Apport de la psychanalyse à la question de l'Addiction. Psychotropes 2008. P. 3-4.

[14] Schäfer M. Le corps comme garant du réel. La lettre de l'enfance et de l'adolescence 2007;69:87-96.

[15] Zapata-Reinet L. Le rapport du sujet au langage : vers une approche des «phénomènes de style » dans la clinique des névroses. L'évolution psychiatrique 2007;72:325-37. 
Tableau 1. - Catégories décelées dans le texte

Categories revealed in the text

\begin{tabular}{|c|c|c|c|}
\hline & Fréquence & Exemples & $\begin{array}{l}\text { Répartition dans le } \\
\text { texte }\end{array}$ \\
\hline \multicolumn{4}{|l|}{ VERBES } \\
\hline factifs & $62,4 \%$ & $\begin{array}{l}\text { Arrive, passait, accroche, } \\
\text { décolle }\end{array}$ & Action \\
\hline statifs & $30,1 \%$ & Venons, soit, brûle, est & Action \\
\hline déclaratifs & $7,5 \%$ & Dis, pense, voit, sais & Action \\
\hline performatif & $0 \%$ & & \\
\hline \multicolumn{4}{|l|}{ CONNECTEURS } \\
\hline addition & $56,7 \%$ & Et, ensuite, puis & Action \\
\hline cause & $13,3 \%$ & Car, alors, donc & Action \\
\hline disjonction & $10,0 \%$ & $\mathrm{Ou}$ & Après-coup \\
\hline opposition & $6,7 \%$ & Mais & Action, Après-coup \\
\hline temps & $6,7 \%$ & Enfin & Mise en place, Action \\
\hline but & $3,3 \%$ & Pour que & Action \\
\hline comparaison & $3,3 \%$ & Comme si & Après-coup \\
\hline condition & $0 \%$ & & \\
\hline lieu & $0 \%$ & & \\
\hline \multicolumn{4}{|c|}{ MODALISATIONS } \\
\hline intensité & $40,5 \%$ & $\begin{array}{l}\text { Un peu, franchement, } \\
\text { quelques, encore }\end{array}$ & Action \\
\hline temps & $21,6 \%$ & $\begin{array}{l}\text { Simultanément, après, } \\
\text { demain }\end{array}$ & Action \\
\hline manière & $16,2 \%$ & Plutôt, à peine, à fond & Action \\
\hline négation & $13,5 \%$ & Pas, ni & $\begin{array}{l}\text { Mise en place, Après- } \\
\text { coup }\end{array}$ \\
\hline lieu & $5,4 \%$ & Près, par terre & Action, Après-coup \\
\hline affirmation & $2,7 \%$ & Aussi & Mise en place \\
\hline doute & $0 \%$ & & \\
\hline \multicolumn{4}{|l|}{ ADJECTIFS } \\
\hline subjectifs & $42,9 \%$ & Inapte, sacré & Après-coup \\
\hline numériques & $38,1 \%$ & & Mise en place, Action \\
\hline objectifs & $19,0 \%$ & Premiers, suivants & Mise en place \\
\hline \multicolumn{4}{|l|}{ PRONOMS } \\
\hline $\mathrm{Je}$ & $74,5 \%$ & & Action \\
\hline on & $13,7 \%$ & & Action \\
\hline Il & $5,9 \%$ & & Mise en place \\
\hline tu & $2,0 \%$ & & Action \\
\hline nous & $0 \%$ & & \\
\hline vous & $0 \%$ & & \\
\hline ils & $0 \%$ & & \\
\hline
\end{tabular}


Tableau 2. - Caractéristiques des épisodes

Characteristics of episodes

\begin{tabular}{llll}
\hline & Episode 1 & Episode 2 & Episode 3 \\
\hline Style & Plutôt énonciatif & Plutôt argumentatif & Plutôt argumentatif \\
Mise en scène & Dynamique, action & Dynamique, action & Dynamique, action \\
Prise en charge & A l'aide du « je » & A l'aide du « je $»$ & A l'aide du « je » \\
\hline
\end{tabular}


Tableau 3. - Répartitions des mots selon les épisodes repérés

Words' distribution according to the episodes identified

\begin{tabular}{|c|c|c|c|}
\hline Catégorie de mots & Episode 1 & Episode 2 & Episode 3 \\
\hline \multicolumn{4}{|l|}{ VERBES } \\
\hline factifs (58) & 12 & 31 & 15 \\
\hline statifs (28) & 5 & 18 & 5 \\
\hline déclaratifs (7) & 0 & 6 & 1 \\
\hline Performatifs (0) & 0 & 0 & 0 \\
\hline \multicolumn{4}{|l|}{ CONNECTEURS } \\
\hline condition & 0 & 0 & 0 \\
\hline addition (17) & 6 & 8 & 3 \\
\hline cause (4) & 0 & 4 & 0 \\
\hline disjonction (3) & 0 & 1 & 2 \\
\hline opposition (2) & 0 & 1 & 1 \\
\hline temps (2) & 1 & 1 & 0 \\
\hline but (1) & 0 & 1 & 0 \\
\hline comparaison (1) & 0 & 0 & 1 \\
\hline Lieu $(0)$ & 0 & 0 & 0 \\
\hline \multicolumn{4}{|l|}{ MODALISATIONS } \\
\hline intensité (15) & 6 & 8 & 1 \\
\hline temps (8) & 2 & 5 & 1 \\
\hline manière (6) & 1 & 3 & 2 \\
\hline négation (5) & 2 & 1 & 2 \\
\hline lieu (2) & 0 & 1 & 1 \\
\hline affirmation (1) & 1 & 0 & 0 \\
\hline Doute (0) & 0 & 0 & 0 \\
\hline \multicolumn{4}{|l|}{ ADJECTIFS } \\
\hline subjectifs (9) & 2 & 3 & 4 \\
\hline numériques (8) & 3 & 3 & 2 \\
\hline objectifs (4) & 3 & $\mathbf{0}$ & 1 \\
\hline \multicolumn{4}{|l|}{ PRONOMS } \\
\hline je (38) & 4 & 24 & 10 \\
\hline on $(7)$ & 3 & 4 & 0 \\
\hline il (3) & 2 & 1 & 0 \\
\hline tu (1) & 0 & 1 & 0 \\
\hline nous (0) & 0 & $\mathbf{0}$ & 0 \\
\hline Vous (0) & 0 & $\mathbf{0}$ & 0 \\
\hline Ils $(0)$ & 0 & 0 & 0 \\
\hline
\end{tabular}


Tableau 4. - Univers de référence

Reference universe

$\begin{array}{llll} & \text { Episode 1 } & \text { Episode 2 } & \text { Episode 3 } \\ \text { Univers 1 } & \text { Sensation (1) Temps } & \text { Corps (2) } & \text { Corps (2) } \\ & (1) & \text { Terre (2) } & \text { Gens (2) } \\ & \text { Gens (1) } & \text { Quantité (2) } & \text { Quantité (1) } \\ & \text { Photographie (1) } & \begin{array}{l}\text { Sensation (1) } \\ \text { Biologie (1) }\end{array} & \text { Terre (1) } \\ & & & \\ \text { Univers 2 } & \text { Gens (1) } & \text { Terre (2) } & \text { Gens (2) } \\ & \text { Sensation (1) } & \text { Pied (2) } & \text { Sang (1) } \\ & \text { Désorganisation (1) } & \text { Tête (1) } & \text { Problème (1) } \\ & \text { Photographie (1) } & \text { Hormones (1) } & \text { Pied (1) } \\ & \text { Temps (1) } & \text { Sensation (1) } & \text { Poids (1) } \\ & & \text { Dimension (1) } & \text { Terre (1) } \\ & & \text { Temps (1) } & \text { Essai (1) } \\ & & \text { Peau (1) } & \\ & & \text { CEil (1) } & \end{array}$

